The title for this Special Section is The Developing Brain: Evidence for Plasticity during Childhood and Adolescence, edited by Amanda E. Guyer, Koraly PerezEdgar, and Eveline A. Crone

\title{
Goal-Directed Correlates and Neurobiological Underpinnings of Adolescent Identity: A Multimethod Multisample Longitudinal Approach
}

\author{
Andrik I. Becht \\ Utrecht University \\ Stefanie A. Nelemans \\ Utrecht University
}

Wilma A.M. Vollebergh, and Susan J.T. Branje
Utrecht University

\author{
Marieke G.N. Bos \\ Leiden University
}

\author{
Sabine Peters \\ Leiden University
}

\author{
Wim H.J. Meeus* \\ Utrecht University and Tilburg University
}

\author{
Eveline A. Crone* \\ Leiden University
}

\begin{abstract}
This multimethod multisample longitudinal study examined how neurological substrates associated with goal directedness and information seeking are related to adolescents' identity. Self-reported data on goal-directedness were collected across three biannual waves in Study 1. Identity was measured one wave later. Study 1 design and measurements were repeated in Study 2 and extended with structural brain data (nucleus accumbens $[\mathrm{NAcc}]$ and prefrontal cortex gray matter volume $[\mathrm{PFC}])$, collected across three biannual waves. Study 1 included 497 adolescents $\left(M_{\text {age T1 }}=13.03\right.$ years $)$ and Study 2 included 131 adolescents $\left(M_{\text {age T1 }}=14.69\right.$ years). Using latent growth curve models, goal directedness, NAcc, and PFC volume predicted a stronger identity one wave later. These findings provide crucial new insights in the underlying neurobiological architecture of identity.
\end{abstract}

Adolescents face the central task of establishing a clear identity (Erikson, 1968) through searching for self-defined values and commitments within various important life domains. This process of

*Shared last authorship.

Data of the Research on Adolescent Development and Relationships (RADAR) study were used. RADAR has been financially supported by main grants from the Netherlands Organisation for Scientific Research (GB-MAGW 480-03-005), and Stichting Achmea Slachtoffer en Samenleving (SASS) to RADAR PI's, and from the Netherlands Organisation for Scientific Research to the Consortium Individual Development (CID; 024.001.003). Data of the Braintime study has been financially supported by a starting grant of the European Research Council (ERC-2010-StG-263234 awarded to Eveline A. Crone), and a grant from the Netherlands Organization for Scientific Research (NWO-VICI 453-14-001 awarded to Eveline A. Crone).

Correspondence concerning this article should be addressed to Andrik I. Becht, Research Centre Adolescent Development, Utrecht University, P.O. Box 80 140, 3508 TC Utrecht, The Netherlands. Electronic mail may be sent to a.i.becht@uu.nl. exploring and reconsidering identity alternatives is assumed to result in strong and stable commitments highly important for psychosocial functioning (Erikson, 1968; Klimstra, Hale, Raaijmakers, Branje, \& Meeus, 2010; Marcia, 1966). For example, adolescents with weaker identity commitments and more uncertainty about their identity commitments show higher levels of internalizing and externalizing problem behaviors over time (e.g., Becht et al., 2016; Crocetti, Klimstra, Keijsers, Hale, \& Meeus, 2009). Thus, development of a strong identity can be considered a crucial task for adolescents' concurrent and future adjustment.

(C) 2018 The Authors

Child Development (c) 2018 Society for Research in Child Development, Inc. All rights reserved. 0009-3920/2018/8903-0012

DOI: $10.1111 /$ cdev. 13048 
An important question concerns why some adolescents develop a strong identity, whereas others do not. One factor that may be particularly important in this regard, but has received little attention in research so far, is the substantial change across adolescence in brain areas involved in behaviors that might facilitate identity formation. Dramatic changes in brain volume are known to occur during adolescence, with protracted development of the prefrontal cortex (PFC; Mills \& Tamnes, 2014) relative to the earlier development of limbic regions, such as the nucleus accumbens (NAcc; Mills, Goddings, Clasen, Giedd, \& Blakemore, 2014). The aim of this multisample longitudinal study was to investigate how identity-relevant behaviors and their neurological substrates relate to subsequent identity in adolescence.

\section{Identity as Goal Directedness and Information Seeking Behavior}

Contemporary models of identity emphasize different dimensions underlying the identity formation process (Crocetti, Rubini, \& Meeus, 2008; Luyckx, Goossens, \& Soenens, 2006). This study used the three-dimensional Meeus-Crocetti model to operationalize management of identity commitments (Crocetti et al., 2008; Meeus, Van De Schoot, Keijsers, Schwartz, \& Branje, 2010). Within this model, commitment refers to strong choices adolescents have made and the certainty they derive from these choices. Reconsideration of commitment represents adolescents' commitment uncertainty and willingness to abandon current commitments to search for new ones. These dimensions define the identity formation cycle in which adolescents form commitments by considering and reconsidering them. Through in-depth exploration, adolescents maintain their current identity commitments by exploring and gathering new information on their commitments cyclically (Crocetti et al., 2008; Meeus et al., 2010).

Based on the Meeus-Crocetti model (Crocetti et al., 2008), establishing a strong identity requires at least two processes involved in identity commitment: goal directedness and information seeking. First, goal directedness and motivation are important capacities needed to pursue valued goals and make commitments (Burrow \& Hill, 2011; Crone \& Dahl, 2012; Erikson, 1968). Indeed, adolescents with strong commitments who explore their commitments to a greater degree demonstrate stronger goal-directed thinking (Burrow, O'Dell, \& Hill, 2010). Moreover, adolescents with stronger feelings of agency report stronger identity commitments
(Schwartz, Côté, \& Arnett, 2005). In addition, adolescents who were actively seeking out identity options and critically evaluating self-relevant information to negotiate identity questions displayed the strongest identity commitments (Berzonsky \& Neimeyer, 1994). Second, information seeking plays a role in identity formation. For example, adolescents with an informational identity style report stronger identity commitments and more active exploration of their commitments in order to strengthen these commitments further (Crocetti, Rubini, Berzonsky, \& Meeus, 2009).

\section{Brain Development and Identity Formation: Goal-Directedness and Information Seeking}

Adolescence is accompanied by structural changes in brain regions critically involved in goal directedness and information seeking behavior (Mills \& Tamnes, 2014). Large-scale changes in PFC and NAcc gray matter volume have been reported with substantial individual differences between adolescents (Gogtay et al., 2004; Mills \& Tamnes, 2014). Concerning the developmental trajectories of NAcc, inconsistent findings have been reported in differentiating left and right NAcc, with some studies reporting decreasing left NAcc (Urošević, Collins, Muetzel, Lim, \& Luciana, 2012), increasing left NAcc but decreasing right NAcc volume (Dennison et al., 2013) across adolescence and young adulthood. A consistent decrease has been reported for bilateral NAcc (e.g., Mills, Goddings, et al., 2014; Østby et al., 2009). However, for both left and right PFC gray matter volume, a consistent decrease in volume has been reported with age (e.g., Mills, Goddings, et al., 2014; Raznahan et al., 2011), which is assumed to reflect decreasing density of synapses and increasing white matter volume to facilitate greater efficiency of information processing (Mills, Goddings, et al., 2014).

Individual differences in level and change observed in subcortical brain regions have also been reported, particularly in the NAcc, which supports reward processing, goal orientation, and motivated behavior. In one of the few longitudinal brain-behavior studies, adolescents with greater versus smaller increases in NAcc volume over time reported more motivation to pursue valued goals (Urošević et al., 2012). Additionally, in a cross-sectional study, higher gray matter volume in NAcc positively related to pursuit of long-term goals (Nemmi, Nymberg, Helander, \& Klingberg, 2016). Because NAcc volume relates to goal directedness, NAcc volume might be involved in identity as well. 
The PFC has been linked to the development of cognitive control and information seeking behavior (Casey, 2015). The PFC shows a protracted development across adolescence (Tamnes et al., 2013), which is assumed to reflect neural plasticity that provides adolescents with opportunities to learn new skills, but also "build an identity" (Blakemore \& Mills, 2014). Thus, although decreasing gray matter volume reflects normative brain maturation, individual differences in its initial level and change over time defines a certain window of opportunity that differs between adolescents. Based on the window of opportunity principle, adolescents who maintain relatively higher PFC volume during adolescence might also reflect more actively on identity issues. Because information seeking behavior is important for exploring and maintaining identity (Berzonsky \& Neimeyer, 1994), we expected that individual differences in the level and changes in PFC structure would predict adolescents' identity as well. To date, most studies have investigated structural development of the NAcc and PFC brain regions separately from behavioral changes (Mills, Goddings, et al., 2014). Yet, individual differences in baseline level of and changes in structure likely relate to behavior (Walhovd, Tamnes, \& Fjell, 2014). In the current longitudinal study, we used a novel approach to test whether the changes in brain areas during adolescence (Mills, Goddings, et al., 2014; Mills et al., 2016) that are involved in observed changes in self-reported goal directedness and information seeking behaviors predict later identity.

\section{This Study}

The purpose of the present research was to investigate self-reported and neural processes underlying adolescents' identity in two separate studies. In Study 1, we tested the hypothesis that adolescents with higher and increasing goal directedness would show a stronger later identity (indicated by relatively high commitments, high indepth exploration and low reconsideration). In Study 2, we drew on questionnaire and neuroimaging data from a second large-scale longitudinal imaging study. First, we aimed to replicate the questionnaire-based findings of Study 1. Second, we examined the general developmental trajectories of NAcc and PFC. On the basis of prior studies, we expected a decrease in PFC brain volume across adolescence (Mills, Goddings, et al., 2014; Raznahan et al., 2011). Because prior studies were inconclusive about change in NAcc volume across adolescence, we could not formulate clear expectations regarding the developmental trajectories of left and right NAcc. Next, we combined questionnaire and imaging data to test whether individual differences in initial level and structural changes of NAcc and PFC gray matter volume predicted later identity commitments, in-depth exploration, and reconsideration of commitments. We hypothesized that a more protracted developmental trajectory in both NAcc and PFC gray matter volume would provide for more opportunities for identity exploration and therefore predict a more mature identity (Erikson, 1968).

\section{Method}

\section{General Methodological and Statistical Approach}

Studies 1 and 2 provided four waves of longitudinal data from two community samples of adolescents. In Study 1, we investigated whether goal-directedness predicted later identity, using self-reports. Similarly, in Study 2 we examined whether self-reported goal directedness predicted later identity. We also investigated whether structural brain changes predicted identity in Study 2. Specifically, we investigated whether initial level and changes in volume of the NAcc and PFC predicted later identity.

To study whether changes in goal directedness (measured with self-reports, and with structural brain measures for areas involved in goal directedness) and information seeking (assessed with structural brain measures) predicted later identity, we conducted a series of latent growth curve models (LGM; Duncan, Duncan, \& Strycker, 2013) on the predictor variables. In both studies, these LGMs were based on three biennial measurement waves (separated by 2-year intervals), covering ages 1121 years across waves (Study 1) and ages 1222 years across waves (Study 2). We assessed adolescents' identity at the fourth wave (see Figure 1 for the conceptual models). LGM is a highly flexible method for analyzing longitudinal data by capturing both the level where individuals start out at the first measurement point (referred to as the initial level or intercept) as well as the change over time from the first to third wave (referred to as the slope). LGM provides estimates of the average intercept and slopes parameters (i.e., fixed effects), which represent the estimates for the entire sample. Individual differences around these overall growth parameters are captured by a variance component (or random effect). LGM allows one to include the intercept and slope estimates to predict the outcome 

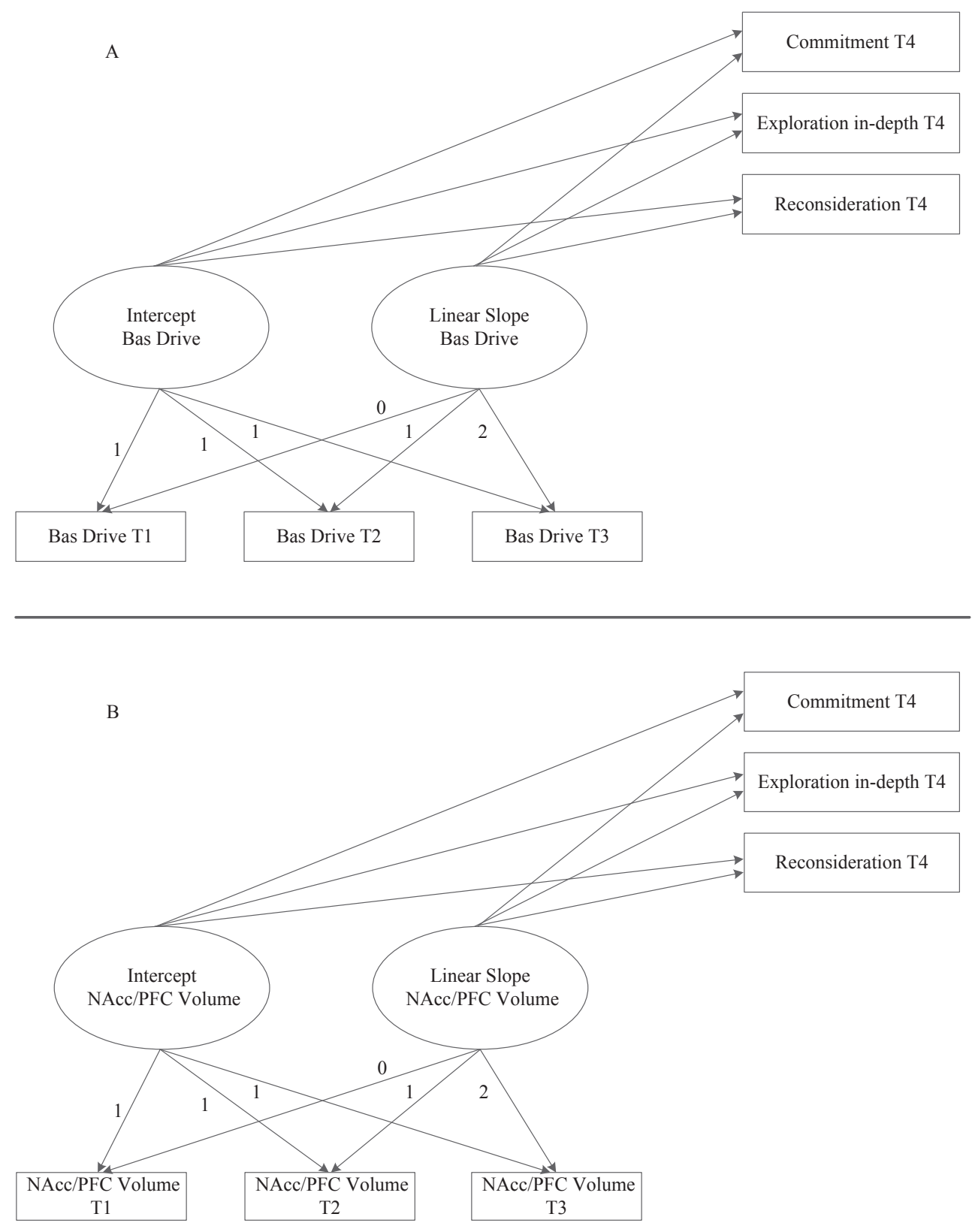

Figure 1. Conceptual latent growth curve model with intercept and slope of (A) BAS drive predicting identity (Study 1 and Study 2), BAS = behavioral activation system, and (B) intercept and slope of NAcC and PFC volume predicting identity (Study 2$)$. NAcc $=$ nucleus accumbens; PFC = prefrontal cortex. Note that separate models were conducted for left NAcc, right, NAcc, left PFC and right PFC.

variable, in our study identity, within the same model (Duncan et al., 2013). That is, we investigated whether adolescents' starting point at the first wave as well as the change over time from Wave 1 one to Wave 3 predicted identity at the fourth measurement wave.

Because adolescents in both studies varied significantly in age at each measurement wave, we applied a model where individually varying times of observations could be estimated (by using the TSCORES option in Mplus, Muthén \& Muthén,
1998-2012). Please find an elaborate description of this TSCORES method as Appendix S1. Given the fact that there were only three measurement waves, we restricted our model to estimate linear slopes only. Because of the multilevel structure when modeling individually varying times of observation (using the TSCORES option in Mplus), standardized coefficients were not available in our prediction models. Therefore, we reported unstandardized coefficients. In all our prediction models, we controlled for gender differences in goal directedness 
and structural brain measures (i.e., NAcc and PFC volume) as well as possible age and gender differences in level of identity commitments, explorationin depth, and reconsideration. If these covariates were, however, nonsignificant, they were trimmed from the models for reasons of parsimony.

\section{Study 1}

\section{Method}

\section{Participants and Procedure}

Participants were 497 Dutch adolescents (283 boys, $\left.M_{\text {age T1 }}=13.03, S D=0.46\right)$ from the ongoing longitudinal project Research on Adolescent Development and Relationships Young Cohort (RADARY). Most adolescents came from medium or high socioeconomic status (SES) families $(87.9 \%)$. For this study, we used data from T1, T3, T5, and T6, which will be designated as Waves 1 through 4, respectively. Wave 1, 2, and 3 were separated by a 2-year interval. Wave 3 and 4 were separated by a 1 -year interval. We chose these measurement waves to match the longitudinal design of Study 2. Adolescents were aged between 11 and 21 years across waves. At Wave 1, adolescents' age ranged between 11.01 and 15.56 years. Adolescents filled out questionnaires during home visits.

Missing value analysis indicated $86 \%$ of all possible data points were completed by the adolescents across waves. Little's missing completely at random (MCAR) test revealed a chi-square $\left(\chi^{2} / d f\right)$ of 1.58 , indicating that is unlikely that results were biased as a result of missing values. Hence, missing data were handled in Mplus 7.4 using full information maximum likelihood (FIML). Participants received $€ 10$ (equivalent to approximately US \$11) when they completed the questionnaires. All participants signed an informed consent form. The medical ethical committee of the University Medical Center Utrecht has approved the RADAR study.

\section{Measures}

Identity. Adolescents reported on their identity using the Utrecht-Management of Identity Commitments Scale (U-MICS; Crocetti et al., 2008). This 26item questionnaire measures identity in three dimensions across educational (13 items) and interpersonal identity domains (13 items). Within the educational and interpersonal domains, the U-MICS measures commitment with five items, exploration in-depth with five items. and reconsideration with three items. Sample items for educational and interpersonal identity are, respectively, "My education/ best friend makes me feel confident about myself" (commitment), "I often think about my education/ best friend" (in-depth exploration), and "In fact, I'm looking for a different education/best friend" (reconsideration), answered on a 5-point Likert scale $(1$ = completely untrue, 5 = completely true). Although the U-MICS allows identity to be measured in different domains, we investigated global identity by combining the educational and interpersonal identity domains in line with other studies (e.g., Crocetti et al., 2008; Meeus et al., 2010). The factorial validity of the U-MICS for the assessment of global identity has been confirmed (Crocetti et al., 2008). Moreover, the U-MICS shows good reliability and measurement invariance across sex and time (Crocetti et al., 2008). Cronbach's alpha at Wave 4 was $.96, .88$, and .91 for commitment, exploration, and reconsideration, respectively.

Goal directedness. Adolescents reported on their goal directedness using a Dutch version of the behavioral activation system (BAS) drive subscale of the behavioral inhibition system/behavioral activation system (BIS/ BAS) questionnaire (Carver \& White, 1994). BAS drive was assessed with four items, for example, "I undertake a lot of effort to get things I want" or "When I want something, I usually go all-out to get it." Items were rated on a 4-point Likert scale $(1=$ strongly disagree, $4=$ strongly agree). Factorial validity of the BAS drive subscale has been established for both the original version (e.g., Carver \& White, 1994) and the translated Dutch version ( $\mathrm{Yu}$, Branje, Keijsers, \& Meeus, 2011). Cronbach's alphas were .58, .67, and .76 for Waves 1 through 3, respectively.

\section{Results}

Means, standard deviations, and correlations among study variables of Study 1 can be found as Appendix S2.

\section{Development of Goal Directedness and Identity}

Parameter estimates of intercept and slope factors of self-reported goal directedness predicting identity are depicted in Table 1. Results indicated that, on average, BAS drive was stable across adolescence, $\quad$ intercept $=12.17, \quad p<.001, \quad \sigma=23.88$, $p=.013$, linear slope $=0.03, p=.436, \sigma=0.12$, $p=.005$. Intercept and slope were negatively correlated, $-1.65, p=.009$, indicating that when adolescents started with relatively higher initial BAS drive level, they reported relatively stronger decreasing 
Table 1

Unstandardized Parameter Estimates and Standard Errors Predicting Identity

\begin{tabular}{|c|c|c|c|c|c|c|}
\hline \multirow[b]{2}{*}{ Predictor } & \multicolumn{2}{|c|}{ Commitment } & \multicolumn{2}{|c|}{ Exploration in-depth } & \multicolumn{2}{|c|}{ Reconsideration } \\
\hline & Parameter & $S E$ & Parameter & $S E$ & Parameter & $S E$ \\
\hline \multicolumn{7}{|c|}{ Study 1: Questionnaire part } \\
\hline BAS drive int. & $0.163^{*}$ & 0.059 & 0.097 & 0.050 & $-0.128^{*}$ & 0.059 \\
\hline BAS drive slope & $2.222^{* *}$ & 0.718 & $1.790^{* *}$ & 0.620 & $-2.070^{*}$ & 0.742 \\
\hline \multicolumn{7}{|c|}{ Study 2: Questionnaire part } \\
\hline BAS drive int. & $0.151^{* * *}$ & 0.009 & $0.117^{* * *}$ & 0.006 & 0.024 & 0.013 \\
\hline BAS drive slope & -0.060 & 0.108 & 0.058 & 0.073 & 0.001 & 0.133 \\
\hline \multicolumn{7}{|l|}{ Study 2 MRI part } \\
\hline Left NAcc int. & $0.110^{* * *}$ & 0.019 & 0.020 & 0.019 & $-0.104^{* *}$ & 0.030 \\
\hline Left NAcc slope & -0.110 & 0.200 & $0.386^{*}$ & 0.187 & 0.156 & 0.313 \\
\hline Right NAcc int. & $0.171^{* * *}$ & 0.015 & 0.025 & 0.018 & -0.016 & 0.026 \\
\hline Right NAcc slope & 0.022 & 0.098 & 0.173 & 0.161 & 0.112 & 0.155 \\
\hline Left PFC int. & 0.071 & 0.160 & 0.171 & 1.266 & 0.092 & 0.415 \\
\hline Left PFC slope & -0.600 & 0.937 & 2.133 & 7.409 & 1.448 & 2.499 \\
\hline Right PFC int. & 0.069 & 0.084 & $0.124^{*}$ & 0.061 & 0.097 & 0.149 \\
\hline Right PFC slope & -0.292 & 0.549 & $1.103^{*}$ & 0.388 & 0.837 & 0.957 \\
\hline
\end{tabular}

Note. In all our prediction models, we controlled for gender differences in intercept (int.) and slope factors as well as possible age and gender differences in identity dimensions. If these covariates were nonsignificant, they were trimmed from the models for reasons of parsimony. $\mathrm{BAS}=$ behavioral activation system; $\mathrm{NAcc}=$ nucleus accumbens; $\mathrm{PFC}=$ prefrontal cortex. ${ }^{*} p<.05 .{ }^{* *} p<.01 .{ }^{* * *} p<.001$.

BAS drive levels over time. Girls reported lower initial levels of BAS drive, $b=-2.87, p=.002$, but a stronger increase in BAS drive over time, $b=.21$, $p=.001$, compared to boys. Consistent with our hypothesis, adolescents with relatively higher initial levels of BAS drive reported stronger commitments and less reconsideration of identity commitments one-wave later (Table 1). Higher baseline levels of BAS drive were not related to exploration in-depth. When adolescents reported a stronger increase in BAS drive they reported stronger commitments, more exploration in-depth and less reconsideration, one wave later.

\section{Discussion}

Findings of Study 1 supported the hypothesis that adolescents who engage in goal-directed efforts (as assessed by BAS drive) were more likely to show stronger identity commitments, more in-depth exploration and less reconsideration of identity commitments at a later moment in time.

\section{Study 2}

To replicate findings of Study 1 and investigate underlying neural mechanisms of identity, we examined a second sample of adolescents.

\section{Method}

\section{Participants and Procedure}

Participants were 138 Dutch adolescents (69 boys, $M_{\text {age }}$ T1 $=14.69$ year, $S D=1.79$ ) who took part in a large longitudinal study named Braintime. The initial sample of Braintime consisted of 299 participants aged between 8 and 25 years at T1 (for details, see Braams, Van Duijvenvoorde, Peper, \& Crone, 2015; Peters, Van Duijvenvoorde, Koolschijn, \& Crone, 2016). For this study, focusing on identity in adolescence, and the validity of our identity measure (i.e., U-MICS; Crocetti et al., 2008) aimed at assessing identity during adolescence and early adulthood, we included a sample of adolescents only. To this end, we selected data for participants who were aged between 12 and 22 years across the three measurement waves, resulting in a sample of 138 adolescents. At T1, these adolescents' age ranged between, 12.05 and 17.91 years. We used questionnaire data (for replication of Study 1) and structural brain data from Waves 1 through 3 with 2-year intervals between assessment waves. Six months later (Wave 4), adolescents' identity was assessed. These measurement points will be further referred to as Waves 1 through 4, respectively. When participants came to the lab for the scan session, they were instructed to lie as still as possible during the whole scan period. They could watch a 
movie of their choice during the high resolution structural scan, which was administered at the end of the scan session. Adolescents received $€ 30$ (equivalent to approximately US \$32) for participation at each time point. Written informed consent for the study (i.e., parental consent and participant assent for adolescents) was provided at each time point. The medical ethical committee of the Leiden Medical Centre approved study procedures. All participants were right handed and reported no neurological or psychiatric impairment at Wave 1. Missing value analyses indicated that $83 \%$ of possible data points were completed by the adolescents across waves. Little's MCAR test revealed a chisquare $\left(\chi^{2} / d f\right)$ of 1.08 , indicating that it is unlikely that results were biased as a result of missing values. Therefore, missing data were handled using FIML.

\section{Measures}

Identity. Similar to Study 1, adolescents reported on their identity at Wave 4 using the U-MICS (Crocetti et al., 2008). Cronbach's alpha was $.88, .76$, and .92 for commitment, exploration in-depth, and reconsideration, respectively.

Goal directedness. As in Study 1, adolescents reported on their goal directedness using the Dutch version of the BAS drive subscale of the BIS/BAS questionnaire (Carver \& White, 1994). Cronbach's alphas were .60, .53, and .72 for Waves 1 through 3, respectively.

Neuroimaging. All participants were scanned on the same 3T MRI scanner (Tesla Philips Achieva MRI system Best, The Netherlands). The longitudinal pipeline in Freesurfer 5.3 (http://surfer.nmr. mgh.harvard.edu/) was used for cortical surface reconstruction and volumetric segregation. Per hemisphere, 31 cortical structures were labeled. All images were visually inspected after processing (longitudinal pipeline) for accuracy (e.g., Mills \& Tamnes, 2014). Poor quality scans were excluded, and high quality scans were reprocessed through the longitudinal pipeline. Note that also single time points were processed longitudinally. We repeated this quality-control procedure until only acceptable scans were included in the processing. No manual editing was performed. From the 404 available scans, we excluded 37 scans. In total, 367 scans were of good quality; 109 participants had scans at three waves, 18 had scans at two waves, 4 had a scan at one wave, and 7 had no available scan of good quality; hence, data of 131 participants were used in the analyses of structural brain data.
Scan acquisition parameters and a detailed description of the structural analyses can be found in Appendix S3.

Regions of interest. We derived the measure of gray matter volume for the NAcc using the volumetric segmentation procedure. Gray matter volume was obtained using the surface-based reconstructed image. PFC was defined by combining the following subdivisions: rostral middle frontal, caudal middle frontal, caudal anterior cingulate, and superior frontal (Mills, Goddings, et al., 2014). We conducted separate analyses for both left and right NAcc and PFC.

\section{Results}

Means, standard deviations, and correlations among Study 2 variables can be found as Appendix S4.

\section{Development of Goal Directedness and Identity}

To replicate Study 1 findings, we investigated whether intercept and linear slope in adolescents' reported BAS drive across three waves predicted identity 6 months later. Parameter estimates of intercept and slope predicting identity are reported in Table 1.

As in Study 1, BAS drive remained stable across adolescence, intercept $=10.93, p<.001, \sigma=3.05$, $p=.052$, linear slope $=0.58, p=.512, \sigma=15.33$, $p=.368$. Intercept and slope were not significantly related, $-4.83, p=.399$, indicating that the rate of change in BAS drive was not associated with the initial level of BAS drive. Boys and girls did not differ on intercept, $b=0.33, p=.597$ and slope, $b=-1.70, p=.344$ of BAS drive. Consistent with our findings from Study 1, adolescents who reported higher initial levels of BAS drive reported relatively stronger commitments. In contrast to Study 1, higher initial levels of BAS drive predicted more exploration in-depth as well. Initial level of BAS drive was not significantly related to adolescents' reconsideration of identity commitments. Also in contrast to Study 1, the linear slope of BAS drive was not related to commitment, exploration in-depth and reconsideration.

\section{Structural Brain Changes and Identity}

To test whether structural brain changes in volume of the NAcc and PFC related to adolescents' identity one wave later, we conducted four separate growth curve models (i.e., a model for left NAcc, a 
model for right NAcc, a model for left PFC, and a model for right PFC). Based on the intercept and linear slope factors of these brain volumes, we predicted adolescents' identity 6 months later. Parameter estimates of NAcc and PFC volume intercept and slope predicting identity are reported in Table 1. The observed individual volume trajectories and average trajectories of left and right NAcc and PFC are presented in Figure 2.

NAcc gray matter volume. Due to previous inconsistency in empirical findings concerning the developmental trajectory of the NAcc, we explored the developmental changes of NAcc volume. We found, that, on average, NAcc volume did not significantly change across adolescence, left NAcc intercept $=5.81, p<.001, \sigma=1.28, p=.546$, linear slope $=-0.43, \quad p=.405, \quad \sigma=0.39 p=.994$, right NAcc intercept $=6.62, p<.001, \sigma=0.89, p=.019$, linear slope $=-0.76, p=.054, \sigma=0.62, p=.884$. Intercept and slope were not significantly related for left NAcc volume, $b=-0.24, p=.984$, and right NAcc volume, $b=-0.51, p=.696$. These findings imply that the relative change in NAcc volume was not associated with the initial level of NAcc volume. Boys and girls did not differ on initial left NAcc volume, $b=0.63, p=.053$ or change over time, $b=-0.18, p=.841$. Yet, boys had higher initial right NAcc volume, $b=0.97, p=<.001$, but a similar rate of change in right NAcc volume, $b=-1.20, p=.090$, relative to girls.

Adolescents with higher initial volume of NAcc (both left and right NAcc) reported stronger
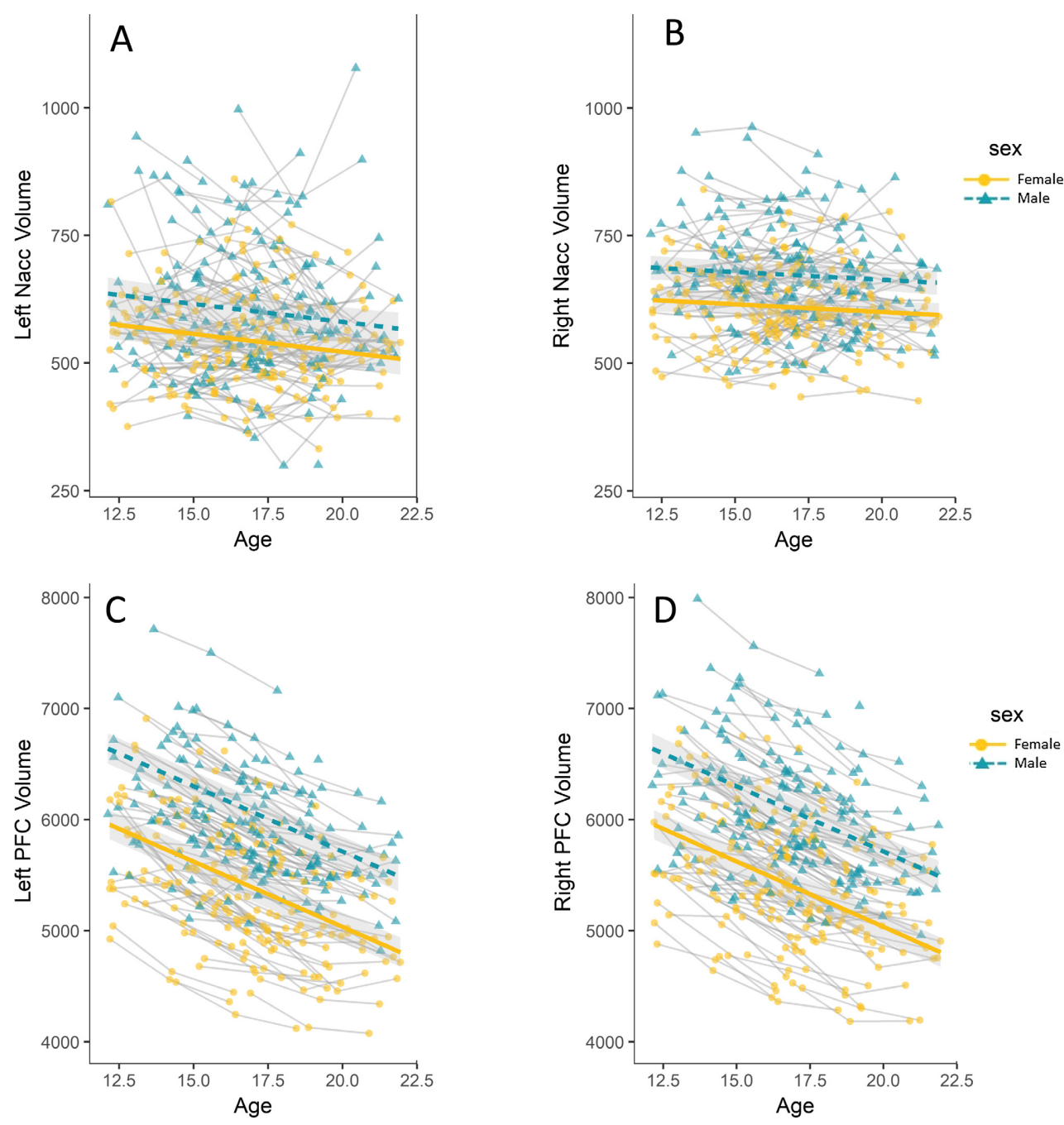

Figure 2. Observed individual volume (in $\mathrm{mm}^{3}$ ) trajectories and average trajectories for (A) Left NAcc, (B) right NAcc, (C) left PFC, and (D) right PFC volume. Shaded areas represent 95\% CI. Raw mean scores for NAcc and PFC volume are displayed. NAcc $=$ nucleus accumbens; $\mathrm{PFC}=$ prefrontal cortex. [Color figure can be viewed at wileyonlinelibrary.com] 
identity commitments at Wave 4. In addition, higher initial volume of the left (but not right) NAcc predicted less identity reconsideration 6 months later. Finally, when adolescents showed a stronger increase in left NAcc volume over time they reported more exploration in-depth 6 months later, but this finding was not found for right NAcc volume.

PFC gray matter volume. PFC volume decreased during adolescence, left PFC intercept $=$ 5.92, $p<.001, \sigma=0.37, p=.002$, linear slope $=-1.01$, $p=.001, \sigma=0.12, p=.927$, right $\mathrm{PFC}$ intercept $=$ 6.00, $p<.001, \sigma=0.43, p<.001$, linear slope $=-0.91$, $p=.006, \sigma=0.18, p=.910$. Intercept and slope were nonsignificantly related for left PFC volume, $b=$ $-0.08, p=.847$, and right PFC volume, $b=-0.11$, $p=.748$. These findings demonstrate that the rate of change in PFC volume was not associated with the initial level of PFC volume. Boys' initial left PFC volume was higher, $b=0.57, p=.001$, as well as boys' initial right PFC volume, $b=0.68, p<.001$. However, the rate of change was similar for boys and girls, for left PFC, $b=-0.19, p=.662$, and right PFC, $b=-0.02, p=.976$.

A higher intercept of right (but not left) PFC volume was related to more exploration in-depth. Moreover, when adolescents showed a weaker decreasing right PFC volume (i.e., they kept relatively higher volume levels across a longer period of time compared to other adolescents), they reported relatively more exploration in-depth 6 months later. However, individual differences in left PFC volume intercept and change were not significantly related to exploration in depth. Hence, findings were not replicated across left and right PFC. Moreover, left and right PFC volume intercept and slope were not related to commitment and reconsideration of identity commitments.

\section{Discussion}

The purpose of Study 2 was to replicate findings based on self-reported goal directedness of Study 1 and to investigate the relationship between structural brain changes and subsequent identity. First, Study 2 partially replicated findings from Study 1, using self-reports, by showing that adolescents' who engaged in goal directedness developed stronger identity commitments and explored these commitments more in depth in order to maintain their commitments. Second, we showed that structural changes in NAcc and PFC during adolescence related to later identity. Specifically, higher baseline differences in both left and right NAcc volume positively predicted later identity commitments. Increase in left NAcc volume over time predicted more in depth exploration, but this finding was not found for right NAcc volume. Furthermore, adolescents with higher initial levels of right (but not left) PFC volume as well as less steeply decreasing right (but not left) PFC volume over time showed more in-depth exploration of their identity commitments.

\section{General Discussion}

This longitudinal multisample study investigated how subcortical and cortical regions involved in goal directedness and information seeking behavior relate to adolescents' identity. Previous studies on adolescents' identity formation suggested the importance of goal directedness and active information seeking to explore, develop, and maintain strong identity commitments (e.g., Burrow et al., 2010; Marcia, 1966; Schwartz et al., 2005). While adolescents' search for identity is accompanied by, and perhaps also facilitated by changes in brain structure, empirical studies investigating structural brain changes and identity are lacking. In this study, we combined a large-scale questionnaire and a structural brain development study. We applied a two-step approach to investigate the longitudinal associations between brain regions, goal directedness, and identity. In the first step, we showed that individual differences in developmental trajectories of selfreported goal directedness predicted later identity. These findings were partially replicated in a second longitudinal adolescent sample. In the second step, we extended these self-reported findings to the neural level by showing how individual differences in initial level and change in NAcc and right PFC gray matter volume predicted adolescents' later identity.

\section{Goal Directedness and Identity}

Our longitudinal self-report findings from Study 1 and Study 2 indicated that adolescents who showed higher initial levels of goal directedness reported relatively stronger identity commitments, explored these commitments more in-depth and were less uncertain about their commitments. We also found that increasing goal directedness over time predicted stronger identity commitments, more in-depth exploration and less reconsideration (e.g., Burrow et al., 2010; Schwartz et al., 2005) but these findings were not replicated in Study 2. Goal directedness might facilitate the identity exploration 
process, which is an important predictor for the formation of identity commitments (Luyckx et al., 2006). For example, when adolescents start questioning and evaluating what type of study or work they want to do later in life, adolescents with a strong goal orientation may be more likely to commit to possible identity choices and to explore them actively to determine what type of study or work suits them. In Study 1 and Study 2 we found that higher initial level in goal directedness positively predicted later identity commitments.

Findings regarding in-depth exploration and reconsideration were not consistent across studies. That is, few associations replicated across Study 1 and Study 2, especially with respect to the linear slopes that were not significant in Study 2. One possible explanation for this discrepancy in findings might result from the difference in sample size between Study 1 and Study 2. Generally, smaller sample sizes tend to produce larger standard errors, resulting in less power to detect a significant effect. Moreover, the larger age heterogeneity at each measurement wave of Study 2 might also have increased the standard errors of the linear slopes, which makes it less likely to identify a significant linear slope. However, the sign of the slopes (indicating negative or positive change) was not always consistent across Study 1 and Study 2 either. Therefore, future studies are needed to replicate our findings. An important question that remains to be answered is the sequence of identity formation processes, which could not be answered in this study because identity was measured only at the latest wave. Future work should further investigate the consecutive steps of identity formation, to see whether changes in goal directedness predict changes in identity exploration, which in turn predict stronger identity commitments. In sum, findings from Study 1 and Study 2 suggested that self-reported goal directedness consistently predicted identity commitments. Findings for exploration in-depth and reconsideration were not consistent across Study 1 and Study 2.

\section{Developmental Trajectories of NAcc Volume, and PFC Volume}

At the neural level, we tested the relationship between trajectories of change in NAcc and PFC volume, on the one hand, and subsequent identity, on the other. First, we found that NAcc (both left and right) gray matter volume remained stable across three waves, which partially replicates previous findings. For example, similar to our findings, right NAcc gray matter volume has been found to be stable across two waves (Urošević et al., 2012), although decreases have been reported as well (Dennison et al., 2013). Prior studies also reported different findings for left NAcc volume development. For example, one study found a decrease during young adulthood across two waves (Urošević et al., 2012), whereas another study reported increased left NAcc across two waves (Dennison et al., 2013).

These discrepancies in findings might have resulted from differences in the age range of participants between studies. With respect to age, the developmental mismatch model would predict that subcortical regions (such as the NAcc) develop earlier relative to cortical regions (Somerville, Jones, \& Casey, 2010). Thus, volume of subcortical regions may show a linear increase early in adolescence, and then stabilize later in adolescence. Consistent with this idea, Urošević et al. (2012) found that left NAcc increased in early adolescence (ages 912 years) but stabilized during late adolescence (1317 years), and decreased in young adulthood. Hence, future studies need to include a broader age range from childhood through young adulthood to further investigate the developmental shape of NAcc. Because the NAcc is a small structure, challenges exist concerning its measurement that might also explain inconsistencies regarding the shape of NAcc volume development (Mills, Goddings, et al., 2014). To further our understanding of developmental trajectories of the NAcc during adolescence, future research should include at least four or more waves of measurement to increase statistical precision (Muthén \& Curran, 1997) and be able to identify the shape of NAcc volume development (i.e., linear, quadratic, and cubic).

Highly consistent findings were observed for PFC over time. In line with prior studies, we found a decrease in PFC gray matter volume across adolescence (e.g., Gogtay et al., 2004; Mills, Goddings, et al., 2014). Changes were highly comparable for left and right PFC volume over time (e.g., Mills, Lalonde, Clasen, Giedd, \& Blakemore, 2014).

\section{Developmental Trajectories of NAcc Volume, PFC Volume, and Identity}

To augment our understanding of the neurobiological processes underlying adolescents' identity, we conducted LGM models to investigate how individual differences in NAcc and PFC volume predicted identity. Results showed that higher levels of left and right NAcc volume were associated with stronger identity commitments 6 months later. In addition, higher levels of left NAcc volume were 
related to less identity reconsideration 6 months later. These brain structure findings from Study 2 add to the self-reported findings from Study 1 and previous questionnaire studies, by showing that goal directedness and its presumed underlying neurological substrates are associated with adolescents' identity formation (e.g., Burrow \& Hill, 2011). Past studies have also found that adolescents with increasing NAcc gray matter volume reported relatively more goal directedness and goal pursuit (Urošević et al., 2012).

Our findings might also further stimulate research on lateralization of the human brain. In general, inconsistent findings in differential functions between the left and right human brain have been reported, except for lateralization for language (Willems, Van der Haegen, Fisher, \& Francks, 2014). For NAcc specifically, it has been found that increase in left (but not right) NAcc was related to higher levels of BAS drive (Urošević et al., 2012). This finding was similar to our finding that left increase in NAcc was related to more in-depth exploration but not right NAcc. Correlations between left and right NAcc ranged between 0.26 and 0.41 , which suggest that left and right NAcc are correlated but also differ substantially. Future studies should further test the unique associations of left and right NAcc and identity formation processes. Our results add to the growing brain-behavior literature by showing that neurobiological underpinnings of goal directedness (i.e., the NAcc) are positively related to adolescents' later commitment making and negatively related to reconsideration of identity commitments.

Together, these findings suggest that different brain regions are involved in different processes of identity formation as described by the identity process model (Crocetti et al., 2008). That is, especially individual differences in initial levels of the left and right NAcc volume were most consistently involved in the process of identity formation, also referred to as the identity formation cycle. In this cycle, adolescents form commitments by considering and reconsidering them (Crocetti et al., 2008; Luyckx et al., 2006). This cycle constitutes two opposing forces of Erikson's (1968) dynamic of identity synthesis (forming commitments) versus identity confusion (questioning identity commitments). The NAcc has been related to goal orientation and motivated behavior (e.g., Urošević et al., 2012). Therefore, individual differences in NAcc volume may especially facilitate the process of pursuing and making certain identity commitments, which is central to the identity formation cycle.
In contrast, individual differences in PFC volume were related to processes involved in the maintenance cycle of identity formation. The PFC has been involved in information seeking and cognitive control (Casey, 2015). Our empirical findings further support this function of the PFC. Specifically, we found that adolescents with greater right PFC volume at baseline were more involved in in-depth exploration of the commitments they already have by actively reflecting on their identity choices, and searching for information about these commitments. Also, adolescents who showed a less steep decrease in their right PFC volume reported higher levels of exploring their commitments in-depth. These findings seem to further confirm the role of the PFC in several behaviors that involve certain levels of cognitive control (Casey, 2015). For example, more PFC gray matter volume has been related to more longterm goal orientation, self-reflection (Blakemore \& Choudhury, 2006), and information seeking behaviors (Casey, 2015). In-depth exploration of identity commitments, involving active reflection upon current commitments, is one such task involving these aspects of cognitive control (e.g., Crocetti et al., 2008; Meeus et al., 2010). Consistent with this role of the PFC, those adolescents with less steeply decreasing PFC volume, and thus relatively more volume across adolescence, were more actively thinking and gathering information about their identity commitments. Our findings that continued higher levels of gray matter volume were related to a stronger identity seem to support a popular neuroscience perspective on adolescent development. According to this perspective, brain changes during adolescence might not only explain increased risk taking but also increasing flexibility to change and adaptation (Casey, 2015; Crone \& Dahl, 2012). The formation of a stable sense of identity can be considered one such complex but important adaptive task in order to prepare for adult roles (Blakemore \& Mills, 2014; Erikson, 1968).

Interestingly, the results showed that developmental changes in gray matter volume occur within relative stability. That is, when adolescents started with higher gray matter volume compared to their peers, most adolescents kept this position across adolescence. As such, these intercept differences might reflect a certain window of opportunity and plasticity for adolescents to develop a strong identity. Specifically, adolescents with higher initial gray matter volume or less steep decreasing gray matter volume, might have a larger window of opportunity to explore their identity compared to adolescents with lower levels of gray matter volume. It 
remains to be empirically tested how changes in brain regions and identity processes influence each other over time. Specifically, an important task for future work is to investigate the temporal order of brain-behavior associations over time.

\section{Strengths, Limitations, and Future Directions}

This study has several important strengths. First, our multisample design allowed us to test our hypotheses in two independent adolescent samples, supporting the replicability of our findings. Second, the longitudinal design of both studies allowed us to investigate how both individual differences at baseline and individual differences in developmental change in goal directedness and structural brain regions related to identity. Third, both studies had a relatively large sample, which increases the accuracy of our estimates. Fourth, this multimethod study examined both self-reported measures and neuroimaging data, an approach selected for furthering understanding of brain-behavior associations (e.g., Mills, Goddings, et al., 2014).

This current study also had some limitations. First, despite longitudinal assessment of NAcc and PFC volumes across three waves, we assessed identity as an outcome at one later time point, prohibiting us from controlling for stability in identity dimensions in previous waves. Future studies should test concurrently ascertained correlated change and bidirectional associations between structural brain volume and identity to investigate possible developmental order. Second, we assessed goaldirectedness at a global rather than a domain specific level, yet levels of goal directedness can vary substantially across different content domains (Deci \& Ryan, 2000). For example, adolescents' drive of doing well in school may be a particularly important predictor of identity commitment making. Third, we only investigated structural differences in NAcc, PFC and identity, whereas profound changes in functional connectivity of subcortical and cortical regions, such as those involved in reward responses (e.g., Van Duijvenvoorde, Peters, Braams, \& Crone, 2016) occur in adolescence. Although we found that structural differences in NAcc predicted adolescents' identity commitment making, some adolescents may experience more feelings of reward when making identity commitments. Future work could test this hypothesis by investigating how individual differences in functional connectivity in reward processing regions relate to adolescents' commitment making. Fourth, we averaged across four subdivisions in the PFC: rostral middle frontal, caudal middle frontal, caudal anterior cingulate and superior frontal, allowing direct comparison with Mills, Goddings, et al. (2014). While our results showed highly consistent developmental trajectories with past work, future work should examine differentiation between PFC subregions. We also focused primarily on lateral PFC given its hypothesized relation with goal directedness, but medial PFC may be more important for internalized deliberation about the self (Crone \& Steinbeis, 2017). Finally, both studies used data from adolescents of relatively high SES families living in The Netherlands. Thus, findings based on self-report measures may not generalize to adolescents living in other countries or from lower SES families.

\section{Conclusion}

This study is one of the first to combine longitudinal assessment of brain development and psychosocial data offering a novel perspective on the neurobiological development underlying adolescents' identity formation. We used a comprehensive assessment of processes related to identity development by relating both the trajectory of goal pursuit as well as NAcc and PFC structural development to adolescents' subsequent identity. Our findings confirmed the hypothesis that adolescents' views about their identity are predicted by the developmental trajectories of level and change in self-reported goal pursuit and structural brain regions. Adolescents with higher goal pursuit and higher NAcc volume reported stronger identity commitments and less uncertainty about these commitments. Moreover, adolescents with higher PFC volume and more protracted PFC volume development reported more reflection on their identity commitments in order to strengthen and maintain them. The current findings are the first evidence of brain-behavior relations involving adolescents' identity formation, and set the stage for future longitudinal studies of linkages between white brain matter and other indices of neural function (e.g., resting state connectivity) on the one hand and identity on the other.

\section{References}

Becht, A. I., Nelemans, S. A., Branje, S. J., Vollebergh, W. A., Koot, H. M., Denissen, J. J., \& Meeus, W. H. (2016). The quest for identity in adolescence: Heterogeneity in daily identity formation and psychosocial adjustment across 5 years. Developmental Psychology, 52, 2010-2021. https: / / doi.org/10.1037/dev0000245 
Berzonsky, M. D., \& Neimeyer, G. J. (1994). Ego identity status and identity processing orientation: The moderating role of commitment. Journal of Research in Personality, 28, 425-435. https:/ / doi.org/10.1006/jrpe.1994.1030

Blakemore, S. J., \& Choudhury, S. (2006). Development of the adolescent brain: Implications for executive function and social cognition. Journal of Child Psychology and Psychiatry, 47, 296-312. https://doi.org/10.1111/j.14697610.2006.01611.x

Blakemore, S. J., \& Mills, K. L. (2014). Is adolescence a sensitive period for sociocultural processing? Annual Review of Psychology, 65, 187-207. https://doi.org/10. 1146/annurev-psych-010213-115202

Braams, B. R., Van Duijvenvoorde, A. C., Peper, J. S., \& Crone, E. A. (2015). Longitudinal changes in adolescent risk-taking: A comprehensive study of neural responses to rewards, pubertal development, and risk-taking behavior. Journal of Neuroscience, 35, 7226-7238. https: / / doi.org/10.1523/jneurosci.4764-14.2015

Burrow, A. L., \& Hill, P. L. (2011). Purpose as a form of identity capital for positive youth adjustment. Developmental Psychology, 47, 1196-1206. https://doi.org/10. $1037 / a 0023818$

Burrow, A. L., O'Dell, A. C., \& Hill, P. L. (2010). Profiles of a developmental asset: Youth purpose as a context for hope and well-being. Journal of Youth and Adolescence, 39, 1265-1273. https:/ / doi.org/10.1007/s10964-009-9481-1

Carver, C. S., \& White, T. L. (1994). Behavioral inhibition, behavioral activation, and affective responses to impending reward and punishment: The BIS/BAS scales. Journal of Personality and Social Psychology, 67, 319-333. https:/ / doi.org/10.1037/ /0022-3514.67.2.319

Casey, B. J. (2015). Beyond simple models of self-control to circuit-based accounts of adolescent behavior. Annual Review of Psychology, 66, 295-319. https://doi.org/10. 1146/annurev-psych-010814-015156

Crocetti, E., Klimstra, T., Keijsers, L., Hale, W. W., \& Meeus, W. (2009). Anxiety trajectories and identity development in adolescence: A five-wave longitudinal study. Journal of Youth and Adolescence, 38, 839-849. https:/ / doi.org/10.1007/s10964-008-9302-y

Crocetti, E., Rubini, M., Berzonsky, M. D., \& Meeus, W. (2009). Brief report: The identity style inventory-validation in Italian adolescents and college students. Journal of Adolescence, 32, 425-433. https://doi.org/10.1016/ j.adolescence.2008.04.002

Crocetti, E., Rubini, M., \& Meeus, W. (2008). Capturing the dynamics of identity formation in various ethnic groups: Development and validation of a three-dimensional model. Journal of Adolescence, 31, 207-222. https:/ / doi.org/10.1016/j.adolescence.2007.09.002

Crone, E. A., \& Dahl, R. E. (2012). Understanding adolescence as a period of social-affective engagement and goal flexibility. Nature Reviews Neuroscience, 13, 636-650. https:/ / doi.org/10.1038/nrn3313

Crone, E. A., \& Steinbeis, N. (2017). Neural perspectives on cognitive control development during childhood and adolescence. Trends in Cognitive Sciences, 21, 205215. https://doi.org/10.1016/j.tics.2017.01.003

Deci, E. L., \& Ryan, R. M. (2000). The" what" and" why" of goal pursuits: Human needs and the self-determination of behavior. Psychological Inquiry, 11, 227-268. https:/ / doi.org/10.1207/s15327965pli1104_01

Dennison, M., Whittle, S., Yücel, M., Vijayakumar, N., Kline, A., Simmons, J., \& Allen, N. B. (2013). Mapping subcortical brain maturation during adolescence: Evidence of hemisphere-and sex-specific longitudinal changes. Developmental Science, 16, 772-791. https://d oi.org/10.1111/desc.12057

Duncan, T. E., Duncan, S. C., \& Strycker, L. A. (2013). An introduction to latent variable growth curve modeling: Concepts, issues, and application. Routledge Academic. https: / /doi.org/10.1111/j.1541-0420.2007.00856_7.x

Erikson, E. (1968). Youth: Identity and crisis. New York, NY: W.W. Norton. https://doi.org/10.1126/science. 161.3838.257

Goddings, A. L., Mills, K. L., Clasen, L. S., Giedd, J. N., Viner, R. M., \& Blakemore, S. J. (2014). The influence of puberty on subcortical brain development. NeuroImage, 88, 242-251. https://doi.org/10.1016/j.neuroimage. 2013.09.073

Gogtay, N., Giedd, J. N., Lusk, L., Hayashi, K. M., Greenstein, D., Vaituzis, A. C., . . Rapoport, J. L. (2004). Dynamic mapping of human cortical development during childhood through early adulthood. Proceedings of the National Academy of Sciences of the United States of America, 101, 8174-8179. https://doi.org/10.1073/pnas. 0402680101

Klimstra, T. A., Hale, W. W., III, Raaijmakers, Q. A., Branje, S. J., \& Meeus, W. H. (2010). Identity formation in adolescence: Change or stability? Journal of Youth and Adolescence, 39, 150-162. https://doi.org/10.1007/ s10964-009-9401-4

Luyckx, K., Goossens, L., \& Soenens, B. (2006). A developmental contextual perspective on identity construction in emerging adulthood: Change dynamics in commitment formation and commitment evaluation. Developmental Psychology, 42, 366-380. https://doi.org/ 10.1037/0012-1649.42.2.366

Marcia, J. E. (1966). Development and validation of egoidentity status. Journal of Personality and Social Psychology, 3, 551-558. https://doi.org/10.1037/h0023281

Meeus, W., Van De Schoot, R., Keijsers, L., Schwartz, S. J., \& Branje, S. (2010). On the progression and stability of adolescent identity formation: A five-wave longitudinal study in early-to-middle and middle-to-late adolescence. Child Development, 81, 1565-1581. https://doi. org/10.1111/j.1467-8624.2010.01492.x

Mehta, P. D., \& West, S. G. (2000). Putting the individual back into individual growth curves. Psychological Methods, 5, 23-43. https: / /doi.org/10.1037//1082-989x.5.1.23

Mills, K. L., Goddings, A. L., Clasen, L. S., Giedd, J. N., \& Blakemore, S. J. (2014). The developmental mismatch in structural brain maturation during adolescence. 
Developmental Neuroscience, 36, 147-160. https://doi. org/10.1159/000362328

Mills, K. L., Goddings, A. L., Herting, M. M., Meuwese, R., Blakemore, S. J., Crone, E. A., ... Tamnes, C. K. (2016). Structural brain development between childhood and adulthood: Convergence across four longitudinal samples. NeuroImage, 141, 273-281. https://doi. org/10.1016/j.neuroimage.2016.07.044

Mills, K. L., Lalonde, F., Clasen, L. S., Giedd, J. N., \& Blakemore, S. J. (2014). Developmental changes in the structure of the social brain in late childhood and adolescence. Social Cognitive and Affective Neuroscience, 9, 123-131. https://doi.org/10.1093/scan/nss113

Mills, K. L., \& Tamnes, C. K. (2014). Methods and considerations for longitudinal structural brain imaging analysis across development. Developmental Cognitive Neuroscience, 9, 172-190. https:/ / doi.org/10.1016/j.dcn.2014.04.004

Muthén, B. O., \& Curran, P. J. (1997). General longitudinal modeling of individual differences in experimental designs: A latent variable framework for analysis and power estimation. Psychological Methods, 2, 371-402. https: / / doi.org/10.1037/1082-989X.2.4.371

Muthén, L. K., \& Muthén, B. O. (1998-2012). Mplus user's guide (7th ed.). Los Angeles, CA: Author.

Nemmi, F., Nymberg, C., Helander, E., \& Klingberg, T. (2016). Grit is associated with structure of nucleus accumbens and gains in cognitive training. Journal of Cognitive Neuroscience, 28, 1688-1699. https://doi.org/ 10.1162/jocn_a_01031

Østby, Y., Tamnes, C. K., Fjell, A. M., Westlye, L. T., Due-Tønnessen, P., \& Walhovd, K. B. (2009). Heterogeneity in subcortical brain development: A structural magnetic resonance imaging study of brain maturation from 8 to 30 years. Journal of Neuroscience, 29, 11772-11782. https://doi.org/10.1523/jneurosci.124209.2009

Peters, S., Van Duijvenvoorde, A. C., Koolschijn, P. C. M., \& Crone, E. A. (2016). Longitudinal development of frontoparietal activity during feedback learning: Contributions of age, performance, working memory and cortical thickness. Developmental Cognitive Neuroscience, 19, 211-222. https://doi.org/10.1016/j.dcn.2016.04.004

Raznahan, A., Shaw, P., Lalonde, F., Stockman, M., Wallace, G. L., Greenstein, D., . . . Giedd, J. N. (2011). How does your cortex grow? Journal of Neuroscience, 31, 7174-7177. https:/ / doi.org/10.1523/jneurosci.0054-11.2011

Schwartz, S. J., Côté, J. E., \& Arnett, J. J. (2005). Identity and agency in emerging adulthood two developmental routes in the individualization process. Youth $\mathcal{E}$ Society, 37, 201-229. https: / / doi.org/10.1177/0044118x05275965

Somerville, L. H., Jones, R. M., \& Casey, B. J. (2010). A time of change: Behavioral and neural correlates of adolescent sensitivity to appetitive and aversive environmental cues. Brain and Cognition, 72, 124-133. https: / / doi.org/10.1016/j.bandc.2009.07.003

Tamnes, C. K., Walhovd, K. B., Grydeland, H., Holland, D., Østby, Y., Dale, A. M., \& Fjell, A. M. (2013). Longitudinal working memory development is related to structural maturation of frontal and parietal cortices. Journal of Cognitive Neuroscience, 25, 1611-1623. https:/ /doi.org/10.1162/jocn_a_00434

Urošević, S., Collins, P., Muetzel, R., Lim, K., \& Luciana, M. (2012). Longitudinal changes in behavioral approach system sensitivity and brain structures involved in reward processing during adolescence. Developmental Psychology, 48, 1488-1500. https://doi.org/10.1037/a00 27502

Van Duijvenvoorde, A. C., Peters, S., Braams, B. R., \& Crone, E. A. (2016). What motivates adolescents? Neural responses to rewards and their influence on adolescents' risk taking, learning, and cognitive control. Neuroscience $\mathcal{E}$ Biobehavioral Reviews, 70, 135-147. https: / / doi.org/10.1016/j.neubiorev.2016.06.037

Walhovd, K. B., Tamnes, C. K., \& Fjell, A. M. (2014). Brain structural maturation and the foundations of cognitive behavioral development. Current Opinion in Neurology, 27, 176-184. https://doi.org/10.1097/wco. 0000000000000074

Willems, R. M., Van der Haegen, L., Fisher, S. E., \& Francks, C. (2014). On the other hand: Including lefthanders in cognitive neuroscience and neurogenetics. Nature Reviews. Neuroscience, 15, 193-201. https://doi. org /10.1038/nrn3679

Yu, R., Branje, S. J., Keijsers, L., \& Meeus, W. H. (2011). Psychometric characteristics of Carver and White's BIS/BAS scales in Dutch adolescents and their mothers. Journal of Personality Assessment, 93, 500-507. https:// doi.org/10.1080/00223891.2011.595745

\section{Supporting Information}

Additional supporting information may be found in the online version of this article at the publisher's website:

Appendix S1. TSCORES option further explained

Appendix S2. Means, Standard Deviations, and Correlations of Behavioral Activation System (BAS) Drive, and Identity (Study 1)

Appendix S3. Technical information scan processing

Appendix S4. Means, Standard Deviations, and Correlations of Behavioral Activation System (BAS) Drive, Nucleus Accumbens (NAcc), Prefrontal Cortex (PFC), and Identity (Study 2) 